\title{
Ações de educação alimentar e nutricional para promoção da saúde de idosos na atenção primária à saúde
}

Food and nutrition education actions to promote the health of the elderly in primary health care

Acciones de educación alimentaria y nutricional para promover la salud de los ancianos en la atención primaria de salud

Débora Suellen Ribeiro Gomes ${ }^{1 *}$, Rejane Maria Sales Cavalcante Mori ${ }^{1}$, Sandra Maria dos Santos Figueiredo $^{2}$, Beatriz Lopes de Oliveira ${ }^{1}$, Julianne Lisboa de Albuquerque ${ }^{1}$, Luana Helena Nogueira Moura ${ }^{1}$, Erica Costa da Costa ${ }^{1}$, Adriene Carvalho da Conceição'.

\section{RESUMO}

Objetivo: Descrever as experiências de atividades de Educação Alimentar e Nutricional (EAN), realizadas com um grupo de idosos em uma Unidade Municipal de Saúde (UMS), em uma cidade no estado do Pará. Relato de experiência: $O$ estudo foi desenvolvido a partir da experiência de acadêmicos do curso de Nutrição de uma universidade pública do Pará no Programa de Educação pelo Trabalho para a Saúde (PET-Saúde/GraduaSUS), que desenvolveram ações de EAN com idosos, de ambos os sexos, frequentadores de um projeto de educação para a saúde em uma UMS, no ano de 2017. As sete atividades realizadas abrangeram as temáticas nutrição, alimentação e saúde com foco nas demandas do público alvo com a utilização de variados recursos didáticos, tais como cartazes, folhetos e folders. Considerações finais: As atividades foram transformadoras, pois houve construção de conhecimento sobre os temas abordados, de forma acessível, incentivando o autocuidado com a saúde por meio do diálogo entre profissional e usuário do sistema público de saúde.

Palavras-chave: Educação em saúde, Alimentação saudável, Atenção à saúde do idoso.

\begin{abstract}
Objective: To describe the experiences of Food and Nutrition Education (ANE) activities, carried out with a group of elderly people in a Municipal Health Unit (UMS), in a city in the state of Pará. Experience report: The study was developed from the experience of academics of the Nutrition course of a public university of Pará in the Program of Education by Work for Health (PET-Saúde/GraduaSUS), who developed ANS actions with the elderly, of both sexes, attending a health education project in a UMS, in 2017. The seven activities covered the themes of nutrition, food and health, focusing on the demands of the target audience with the use of various teaching resources, such as posters, brochures and folders. Final considerations: The activities were transformative, because there was a construction of knowledge about the topics addressed, in an accessible way, encouraging self-care with health through the dialogue between professional and user of the public health system.
\end{abstract}

Key words: Health education, Healthy eating, Elderly health care.

${ }^{1}$ Universidade Federal do Pará (UFPA), Belém - PA. *E-mail: nutdeboraribeiro@gmail.com

${ }^{2}$ Secretaria Municipal de Saúde de Belém (SESMA), Belém - PA.

SUBMETIDO EM: 10/2021

ACEITO EM: 11/2021

PUBLICADO EM: 12/2021 


\section{RESUMEN}

Objetivo: Describir las experiencias de las actividades de Educación Alimentaria y Nutricional (ANE), realizadas con un grupo de personas mayores en una Unidad Municipal de Salud (UMS), en una ciudad del estado de Pará. Informe de experiencia: El estudio fue desarrollado a partir de la experiencia de académicos del curso de Nutrición de una universidad pública de Pará en el Programa de Educación por trabajo para la salud (PET-Saúde/GraduaSUS), quienes desarrollaron acciones de SNA con ancianos, de ambos sexos, asistiendo a un proyecto de educación para la salud en una UMS, en 2017. Las siete actividades abarcaron los temas de nutrición, alimentación y salud, centrándose en las demandas del público objetivo con el uso de diversos recursos didácticos, como carteles, folletos y carpetas. Consideraciones finales: Las actividades fueron transformadoras, porque hubo una construcción de conocimiento sobre los temas abordados, de manera accesible, fomentando el autocuidado con salud a través del diálogo entre profesional y usuario del sistema público de salud.

Palabras clave: Educación para la salud, Alimentación saludable, Cuidado de la salud para personas mayores.

\section{INTRODUÇÃO}

A Pesquisa Nacional por Amostra de Domicílios Contínua (PNAD), divulgada pelo Instituto Brasileiro de Geografia e Estatística (IBGE), mostrou que no Brasil, entre 2012 e 2019, foi mantido o alargamento do topo e o estreitamento da base da estrutura etária do país, evidenciando o envelhecimento populacional, na qual, entre a população total estimada em 2019 de 209,496 milhões de pessoas, 15,7\%, cerca de 32,860 milhões, eram idosos com 60 anos ou mais (IBGE, 2020).

O envelhecimento é um processo natural, complexo e contínuo, influenciado por aspectos culturais, econômicos, epidemiológicos e subjetivos, determinando transformações no indivíduo como perda da capacidade motora e funcional, fragilidade, déficits de cognição e presença de doenças crônicas, além disso há menor disponibilidade de recursos sociais e financeiros, tanto no nível individual quanto da sociedade, o que demanda maior atenção quando se fala em garantia da saúde (SARAIVA LB, et al., 2017; VERAS RP e OLIVEIRA M, 2018; SCIAMA DS, et al., 2020).

Outro fator que incide sobre a saúde do idoso é o excesso de peso e obesidade, que provocam doenças de alta morbimortalidade como diabetes mellitus (DM), hipertensão e doenças cardiovasculares, condições crônicas que costumam ser mais expressivas quanto maior for a idade do indivíduo e revelam a necessidade de ações multiprofissionais e interdisciplinares para promover, de forma integrada, conhecimento acerca do envelhecimento e atenção efetiva e eficaz a sua saúde (KOLITSKI MF, et al., 2019; MAEYAMA MA, et al., 2020; HADDAD PCMB e CALAMITA Z, 2020).

Placideli N, et al. (2020) destaca que os serviços da Atenção Primária à Saúde (APS) se mostram eficazes em tratar as doenças crônicas e seus agravos, entretanto, atividades para prevenção e promoção da saúde se mostram ineficientes, com inexistência de trabalho conjunto que facilitaria 0 atendimento integral do idoso, evidenciando a forte associação entre longevidade e o desenvolvimento de doenças crônicas, mas ressalta que não se deve reduzir a velhice ao conjunto de enfermidades, portanto é essencial o planejamento de ações preventivas e integrais.

Com relação a nutrição do idoso, mudanças fisiológicas e o uso regular de medicamentos influenciam o paladar, ingestão e digestão dos alimentos e absorção de nutrientes necessários para manutenção de um estado nutricional adequado, assim, têm-se no cuidado com a alimentação uma das estratégias mais importantes para promover saúde e evitar diversos agravos decorrentes de hábitos de vida pouco ou não saudáveis, compreendendo-se que mesmo quando a autonomia e capacidades estão comprometidas, a velhice pode ser pensada como uma fase ativa, se bem assistida (OLIVEIRA HCC, et al., 2017; ABREU BM, et al., 2018; BOLICO PFA, et al., 2021; PASSOS ACM, et al., 2021). 
Portanto, este trabalho tem como objetivo relatar experiências de atividades de Educação Alimentar e Nutricional (EAN), direcionadas à idosos que frequentam reuniões semanais em uma Unidade Municipal de Saúde (UMS) Estado do Pará, como forma de compartilhar vivências e demonstrar a essencialidade e aplicabilidade de ações educativas planejadas e executadas com este público, com o intuito de contribuir para sua qualidade de vida.

\section{RELATO DE EXPERIÊNCIA}

Trata-se de um relato de experiência de atividades de Educação Alimentar e Nutricional (EAN), realizadas com um grupo de idosos de uma Unidade Municipal de Saúde (UMS) no Pará, durante o ano de 2017, executadas pela equipe do Programa de Educação pelo Trabalho para a Saúde (PETSaúde/GraduaSUS) de uma Universidade Pública do Estado e Secretaria Municipal de Saúde.

Para integrar a equipe de pesquisadores, o PET-Saúde/GraduaSUS contou com um tutor, professor titular de uma universidade pública do Pará e preceptor, nutricionista vinculado à Secretaria Municipal de Saúde com atuação na UMS em que o projeto ocorreu, além de acadêmicas do curso de nutrição.

Os participantes alvo do projeto de Educação Alimentar e Nutricional (EAN) a qual foi obtido o relato dessa experiência se tratavam de idosos, de ambos os sexos, matriculados na unidade e dirigidos pela assistente social, que promove reuniões semanais para a participação de atividades educativas e multiprofissionais, como palestras, rodas de conversa, oficinas e dança, contando com o apoio de uma equipe multidisciplinar, incluindo a nutrição.

Para a elaboração do plano de EAN, foi inicialmente realizado um encontro com os idosos, no qual foram sugeridos e escolhidos temas sobre alimentação e nutrição para serem abordados. Os encontros aconteceram mensalmente, com duração média de 1 hora, utilizando diversos materiais didáticos como cartazes, painéis, folder, livretos e folhetos e tinham o intuito de estimular as discussões acerca da temática.

Foram pensadas temáticas para 7 encontros com o grupo, todas relacionadas às demandas que 0 público alvo trazia para as reuniões anteriores, o que tornou as dinâmicas mais significativas. A cada semana, uma abordagem diferente era trabalhada em grupo, começando pela "Sensibilização sobre o cuidado com sua saúde a partir da alimentação", "Você é o que você come", "Arraial saudável", "Cuidados com a hidratação", "Almoço do Círio de Nazaré", "Falando sobre os adoçantes" e, por fim, "Aproveitamento integral dos alimentos". A seguir consta a descrição de cada atividade realizada.

A primeira abordagem, cujo o tema é "Sensibilização sobre o cuidado com sua saúde a partir da alimentação" tinha o objetivo de falar sobre a saúde do idoso de forma lúdica, deixando as participantes a vontade para expressar dificuldades. Cada participante via sua imagem através de um espelho dentro de uma caixa e falava uma característica alcançada e outra que precisava mudar ou melhorar no cuidado com a saúde, especialmente no que se refere a alimentação.

Em seguida, foi realizada uma dinâmica; em duplas as idosas estouravam um balão e dele era retirado um papel com nome de uma fruta; para cada alimento foi feito um relato sobre seu valor nutricional e os benefícios de sua inclusão na alimentação diária.

A segunda atividade nomeada "Você é o que você come" teve a finalidade de relacionar a influência que os sentimentos exercem na alimentação. Inicialmente, através de uma exposição dialogada, tendo como ponto de partida o relato das idosas sobre os seus hábitos alimentares, foi feita uma relação da importância dos mesmos com suas emoções e seus sentimentos, os quais atuam direta nas suas escolhas alimentares, para que entendessem a diferença entre o "querer comer" e o "ter fome". Isso gerou questionamento, interpretação e discussão a partir do reconhecimento e confronto com a realidade, favorecendo a análise crítica e produção de novos conhecimentos.

Já a terceira ação "Arraial saudável", visou promover práticas alimentares adequadas e incentivar o consumo de pratos típicos juninos mais saudáveis. Foi apresentada a possibilidade de aproveitar as festas juninas com uma alimentação mais equilibrada. O planejamento da atividade consistiu na elaboração de um caderno de receitas típicas da época com teor reduzido de sódio, açúcares, gorduras e de baixo custo. 
Durante a experiência foi feita a degustação de um bolo de fubá light e todos os idosos receberam um caderno de receitas típicas da época junina, contendo informações nutricionais e custo de preparação. Ao final foi aberta a oportunidade para perguntas e respostas, para esclarecer e avaliar o grau de entendimento dos participantes.

A quarta abordagem foi relacionada aos "Cuidados com a hidratação", na qual foram demonstrados os benefícios de uma boa hidratação para a manutenção das funções orgânicas dos indivíduos. O grupo foi alertado para as mudanças que esta fase da vida traz, com ênfase para a maior necessidade de ingestão de água em detrimento à redução da sensação de sede. Com auxílio de placas sobre os benefícios da água para a saúde, foram questionados sobre hábitos diários com relação a hidratação e qualidade da água que consomem, inclusive no preparo dos alimentos.

No decorrer do diálogo com os participantes, informações essenciais sobre a compra, armazenamento e ingestão segura da água foram apresentadas. Em seguida algumas perguntas foram feitas, como forma de identificar o nível de entendimento dos idosos sobre o assunto abordado e, por fim, foi entregue para cada idoso uma amostra de hipoclorito de sódio a 2,5\% para purificar a água e higienizar os alimentos.

A quinta ação referiu-se ao "Almoço do Círio de Nazaré", com o objetivo de avaliar o grau de conhecimento das idosas sobre a informação nutricional dos pratos típicos paraenses consumidos na época do Círio de Nossa Senhora de Nazaré. As informações foram apresentadas em quadros que continham: imagem da preparação, porção recomendada em medidas caseiras e em gramas, valores nutricionais para macronutrientes, fibras, calorias e sódio em gramas e miligramas de pratos tradicionais da cultura paraense (pato no tucupi, maniçoba e vatapá); a ação permitiu que os usuários opinassem sobre a porção que julgavam adequada e seu respectivo valor calórico.

A sexta atividade foi "Falando sobre os adoçantes", com o intuito de orientar sobre aspectos que envolvem o consumo indiscriminado de adoçantes e seus riscos. Foi desenvolvida em três momentos: 0 primeiro com perguntas a respeito dos adoçantes que eram consumidos pelos participantes, apresentação de cada um com suas vantagens, desvantagens, indicações, contraindicações e com esclarecimentos sobre as principais marcas comercializadas.

O segundo momento foi dedicado ao diálogo com os idosos, com exposição de relatos sobre seu consumo de adoçantes, quantidade ingerida, tempo de uso e se houve prescrição e/ou orientação médica/nutricional para o consumo. No terceiro e último momento houve degustação de um bolo diet e de um suco de fruta, ambos preparados com adoçante natural. Para finalizar, foram entregues materiais educativos, contendo informações sobre o tema abordado e receitas elaboradas com o uso de adoçantes.

A sétima foi sobre o "Aproveitamento integral dos alimentos", com o propósito de orientar e incentivar o aproveitamento integral dos alimentos (AIA), como alternativa de alimentação nutritiva e de baixo custo. Na atividade foi verificado o nível de conhecimento dos participantes sobre o AIA e, por meio de uma roda de conversa a respeito da importância desta técnica, foram tratados seus benefícios ambientais e nutricionais e exposição de alimentos da culinária paraense que podem ser usados nem sua totalidade, agregando alto valor nutricional às preparações.

Após isso, os participantes degustaram um bolo de casca de banana e, através de uma ficha com escala hedônica facial, avaliou-se o nível de aceitabilidade para tal preparação. Nesta avaliação o idoso votava no "gostei extremamente", se a preparação estava ótima; "gostei moderadamente", se a preparação estava boa; "nem gostei/nem desgostei", se a preparação estava regular; no "desgostei moderadamente", se a preparação estava ruim e "desgostei extremamente", se a preparação estava muito ruim. Ao final, as idosas receberam uma cartilha educativa com informações sobre o tema abordado e a receita degustada.

\section{DISCUSSÃO}

A expressiva participação dos idosos nas atividades, a fácil interação, disponibilidade em ouvir os relatos do cotidiano, a desmistificação de conceitos incoerentes sobre os temas abordados, a identificação do 
entendimento acerca das principais orientações para o autocuidado através das dinâmicas e o interesse pelo material educativo entregue foram resultados observados, o que comprovou a importância de atividades educativas para promoção da saúde na Atenção Básica e a aplicabilidade desse tipo de ação na área da alimentação e nutrição para melhorar a qualidade de vida deste público.

A experiência possibilitou constatar que muitos participantes não detinham conhecimentos básicos sobre os assuntos abordados de forma que servissem para o autocuidado. No geral, não existia muita clareza sobre a influência da alimentação saudável para a saúde, assim como as implicações negativas de más escolhas alimentares, o que corrobora com as afirmações de Silva, et al. (2017) a respeito da importância da extensão universitária com este grupo etário para permitir o redimensionando das técnicas de cuidado, enfatizando a relação teoria-prática, priorizando a condição de saúde em questão.

Proporcionar esse aprendizado foi uma forma de garantir um direito que é a assistência à saúde no SUS para prevenir, promover, proteger e recuperar a saúde, além de reafirmar o valor da educação em saúde de forma dialógica para este público, elevando seu poder de compreensão de si e de sua realidade (BRASIL, 1994; BOLICO PFA, et al., 2021).

Além disso, o cuidado ao idoso na APS precisa superar o manejo fragmentado, focado somente na doença, com consultas especializadas e isoladas, informações pouco ou não compartilhadas, inúmeras prescrições de medicamentos e solicitações de exames e distanciamento da realidade do usuário, do contrário, o cuidado à pessoa idosa deve contar com atividades participativas e de grupos e de forma multiprofissional para assegurar um envelhecimento ativo e saudável (MINISTÉRIO DA SAÚDE, 2006; VERAS RP e OLIVEIRA M, 2018; SCIAMA DS, et al., 2020; SOUZA EM, et al., 2021).

As dinâmicas realizadas estabeleciam um vínculo de proximidade e confiança entre os idosos e as acadêmicas, o que culminava em trocas de conhecimentos, com respostas a perguntas, reflexão sobre questionamentos e atitudes, revelando o ambiente confortável para a promoção da saúde, conforme o ministério da saúde (2006) e Veras RP e Oliveira M (2018), sobre o trabalho em grupo com idosos que amplia sua relação com o profissional da saúde, criando um espaço que vai além da consulta.

Intervenções sociais e comportamentais são, ainda, potencialmente influenciadoras de mudanças no comportamento do indivíduo, provocando alterações no hábito alimentar e estado nutricional e cabe ao nutricionista contribuir com orientações sobre o preparo e seleção dos alimentos, incentivo a realização das refeições coletivas e preservação de habilidades do idoso, com estímulo ao desenvolvimento de novas, considerando seus saberes prévios, valores, desejos e interesses (PADILHA RQ, 2018; SEABRA CAM, et al., 2019).

É importante ressaltar a essencialidade da qualificação profissional que preconiza a humanização e a integralidade do cuidado ao idoso na APS, pois desta maneira será mais fácil aproximá-lo dos programas que são desenvolvidos para eles, garantindo um espaço que vai além da consulta e visa seu bem estar físico, social e mental, incentivando cada vez mais sua busca pelos serviços e autocuidado (NASCIMENTO GJLP, et al., 2020).

As atividades de EAN realizadas com esse grupo foram construtivas e transformadoras, possibilitaram a propagação de informações cientificamente comprovadas sobre diversos temas da saúde do idoso de forma interativa, dinâmica e acessível, a fim de contribuir para o conhecimento e sensibilização com relação à prevenção, identificação, tratamento e/ou recuperação de agravos comuns neste público, atendendo às necessidades identificadas.

\section{AGRADECIMENTOS E FINANCIAMENTO}

Universidade Federal do Pará (UFPA); Unidade Municipal de Saúde do Guamá; Secretaria Municipal de Saúde (Sesma), PET - Saúde/GraduaSUS, nutricionistas responsáveis e assistente social que geraram oportunidades que contribuíram diretamente para a formação acadêmica, profissional e pessoal das alunas, colaborando assim para a produção do artigo. Todos os autores aprovaram a versão final para publicação. 


\section{REFERÊNCIAS}

1. ABREU BM, et al. Envelhecimento Ativo: das diretrizes às ações para melhorar a qualidade de vida das pessoas idosas. Perspectivas em Políticas Públicas. Belo Horizonte, 2018; 11(21): 129-172.

2. BRASIL. Lei $n^{\circ} 8.842$, de 4 de janeiro de 1994. Dispõe sobre a Política Nacional do Idoso, cria o Conselho Nacional do Idoso e dá outras providências. Disponível em: http://www.planalto.gov.br/ccivil_03/leis/l8842.htm. Acesso: 22/04/2021

3. BRASIL. Portaria no 2.528 de outubro de 2006. Aprova a Política Nacional de Saúde da Pessoa Idosa. Disponível em: https://bvsms.saude.gov.br/bvs/saudelegis/gm/2006/prt2528_19_10_2006.html. Acesso: 22/11/2020.

4. BOLICO PFA, et al. Ação educativa sobre alimentação saudável para um grupo de idosos. Brazilian Journal of Health Review, 2021; 4(2): 7481-7491.

5. CARDOSO RSS, et al. Tecnologia educacional: um instrumento dinamizador do cuidado com idosos. Revista Brasileira de Enfermagem, 2018; 71(2): 839-45.

6. FRANÇA CJ, CARVALHO VCHS. Estratégias de educação alimentar e nutricional na Atenção Primária à Saúde: uma revisão de literatura. Saúde Debate [online], 2017; 41(114): 932-948.

7. HADDAD PCMB, CALAMITA Z. Aspectos sociodemográficos, qualidade de vida e saúde do idoso institucionalizado. Revista de enfermagem UFPE on line, 2020; 14: e243416.

8. INSTITUTO BRASILEIRO DE GEOGRAFIA E ESTATÍSTICA (IBGE). Pesquisa Nacional por Amostra de Domicílios Contínua: Características gerais dos domicílios e dos moradores. 2019. Rio de Janeiro. 2020. Disponível em: https://biblioteca.ibge.gov.br/visualizacao/livros/liv101707_informativo.pdf. Acesso: 03/12/2020.

9. KOLITSKI MF, et al. Associação entre o nível glicêmico, risco cardiovascular e qualidade de vida em idosos diabéticos. Revista Saúde e Desenvolvimento Humano, 2019; 7(3):1-6.

10. MAEYAMA MA, et al. Saúde do Idoso e os atributos da Atenção Básica à Saúde. Brazilian Journal of Development, Curitiba, 2020; 6(8): 55018-55036.

11. MINISTÉRIO DA SAÚDE. Secretaria de Atenção à Saúde, Departamento de Atenção Básica. Envelhecimento e saúde da pessoa idosa: Cadernos de Atenção Básica - n. .019

Série A. Normas e Manuais Técnicos Brasília - DF: Brasil, 2006. Disponível em: https://bvsms.saude.gov.br/bvs/publicacoes/evelhecimento_saude_pessoa_idosa.pdf. Acesso em: 22/04/2021.

12. NASCIMENTO GJLP, et al. A Importância Da Humanização No Atendimento Ao Idoso Na Atenção Básica: Revisão Bibliográfica. Revista de Iniciação Científica e Extensão, 2020; 3(2):472-82.

13. OLIVEIRA, HCC et al. Alimentação e nutrição dos idosos: uma revisão bibliográfica. In: Anais do Congresso Internacional de Enfermagem. Universidade Tiradentes. 2017.

14. PADILHA RQ, et al. Princípios para a gestão da clínica: conectando gestão, atenção à saúde e educação na saúde. Ciência \& saúde coletiva, Rio de Janeiro, 2018; 23(12): 4249-4257.

15. PASSOS ACM, et al. Qualidade da alimentação de idosos longevos e doenças crônicas não transmissíveis. Semina: Ciências Biológicas e da Saúde, Londrina, 2021; 42(2): 167-178.

16. PLACIDELI N, et al. Avaliação da saúde do idoso na atenção primária. Revista de Saúde Pública, 2020; 54(6).

17. SARAIVA LB, et al. Avaliação Geriátrica Ampla e sua Utilização no Cuidado de Enfermagem a Pessoas Idosas. Journal Health Science, 2017; 19(4): 262-267.

18. SEABRA CAM, et al. Educação em saúde como estratégia para promoção da saúde dos idosos: Uma revisão integrativa. Revista Brasileira de Geriatria e Gerontologia, 2019; 22(4): 2-12.

19. SCIAMA DS, et al. Active ageing: social representations of health professionals in Elderly Health Reference Units. Revista da Escola de Enfermagem da USP. 2020; 54:e03605.

20. SILVA W, et al. Ações educativas vivenciadas com idosos: um relato de experiência. Revista de Ciências da Saúde Nova Esperança, 2017; 15(3): 2317-7160.

21. SOUZA EM, et al. Educação popular, promoção da saúde e envelhecimento ativo: uma revisão bibliográfica integrativa. Ciência \& Saúde Coletiva, 2021; 26(4):1355-1368.

22. VERAS RP; OLIVEIRA M. Envelhecer no Brasil: a construção de um modelo de cuidado. Ciência \& Saúde coletiva [online]. 2018; 23(6): 1929-1936. 